\title{
A theoretical study of the kernel function for resistivity prospecting with a Schlumberger apparatus in water $(*)$
}

\author{
D. Patella $(* *)-$ D. Schiavone $(* * *)$
}

Received on April 29th, 1974

Sumary. - On the basis of already known theoretical results concerning the use of the greoelectrical sounding metlod with the apparatus immersed in water, in the present paper we individuate the kernel function of the particular geometry of the problem. The study of the properties of the kernel function allows a better understanding of the possibilities of application of the new method. The theoretical results that we deseribe must be regarded, among other things, as the basis for the derivation of a direct quantitative interpretation method.

Riassunto. - Sulla base di risultati teorici gia noti circa l'uso del metodo del sondaggio geoelettrico con il dispositivo elettrodico immerso in accua, nel presente lavoro viene individuata la funzione nucleo della particolare geometria del problema. Lo studio delle proprieta della funzione nucleo permette una migliore comprensione delle possibilità di applicazione del nuovo metorlo. I risultati teorici che descriviamo debbono intendersi, tra laltro, come la base per ricavare un metodo diretto di interpretazione quantitativa.

(*) Paper read at the 3rd Neeting of the "Associazione Geofisica Italiana "- Rome, April 18-20, 1974.

(**) Osservatorio di Geofisica e Fisica Cosmica - University of Bari, Italy.

(***) Istituto di Geodesia e Geofisica - University of Bari, Italy. 


\section{1. - Introduction}

Since many years ago it has been theoretically and experimentally demonstrated by some russian geophysicists $(4,5)$ the possibility of carrying out resistivity soundings on the sea or lakes with the electrode array immersed in water with the aim of giving useful contributions to the solution of geological-structural, geological-technical and petroleum prospecting problems. The reason why such technique has been preferred to the usual one with the apparatus on the free surface is that very often with the latter technique the presence of relatively thin layers at or near the bottom of the water can be hardly localized or even masked by the water layer.

Terekhin (') derives the general expression of the apparent resiistivity for the Schlumberger and dipole axial arrays with the apparatus at the bottom of the water layer. On the basis of such theory a catalogue of master curves has been prepared for two and three-layer models which is actually hardly available.

In the present work we will show how it is possible, in accordance with the analogous problem for normal soundings, to derive a kernel function which has the following three important properties:

a) it is mathematically simple and easily handled, so allowing to derive all the electrical properties of a multilayer section in a faster manner than the apparent resistivity function;

b) it is possible to make an immediate comparison with the kernel function for the apparatus at the air-water boundary with the aim of showing the differences between the two functions with particular attention to the resolving power;

c) a complete study of all the properties of the new kernel function does constitute the basis to the derivation of a direct quantitative interpretation method that will be the argument for a next paper.

\section{2. - General expression of the Schlumberger apparent Resisti- VITX WI'II TILE APPARATUS AT TILE BOTTOM}

Let us consider an homogeneous horizontally $n$-stratified section whose upper layer is the water layer (see fig. 1$)$. The $i$-th layer $(i=1$, $2, \ldots, n-1, n)$ is identified by its thickness $h_{i}$ and resistivity $\varrho_{i}$. 
The point current source $A$ of strength $+I$ is placed in the water layer in general at a depth $z_{0} \leqslant h_{1}$ from the surface.

Assuming a cylindrical system of coordinates $(r, \vartheta, z)$ with the origin at the point $A$ and the $z$-axis vertically downward, the potential $U_{i}$ at a point $M$ of the $i$-th layer, distant $R$ from $A$, can be thought

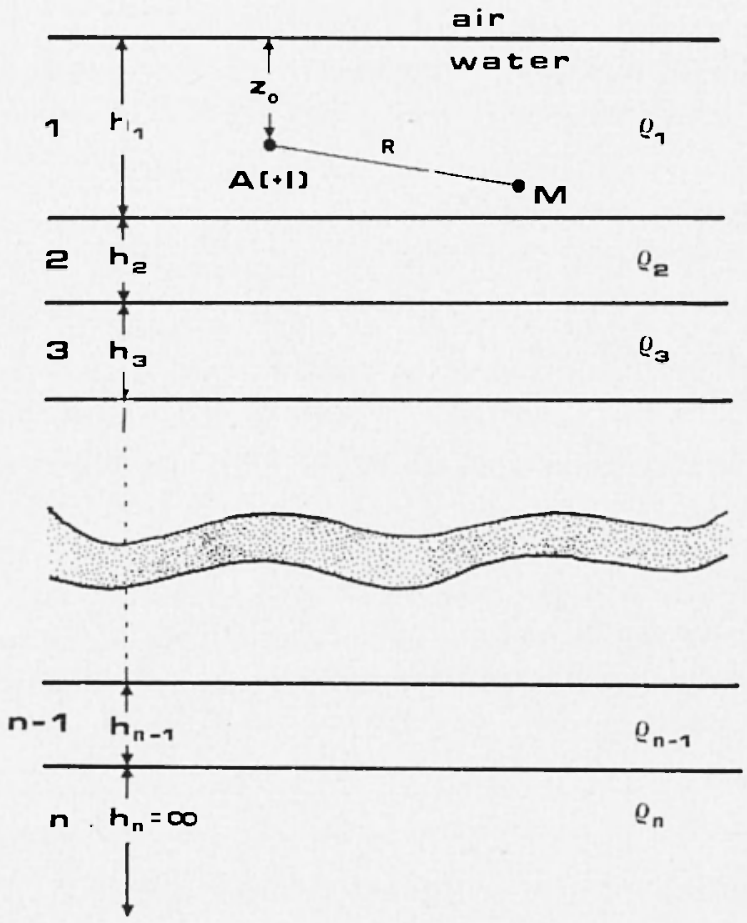

Fig. 1 - Depiction of a geoelectrical n-layered section.

of as a sum of a normal potential $V=I_{Q_{1}} / 4 \pi R$ which would be measured in a conducting space with resistivity $\varrho_{1}$, plus a disturbing potential $U^{\prime}{ }_{i}$, caused by the boundaries, which, owing to the axial symmetry of the problem with respect to the $z$-axis, is a solution of the following explicit form of the Laplace equation

$$
\frac{\partial^{2} U_{i}^{\prime}}{\partial r^{2}}+\frac{1}{r} \frac{\partial U_{i}^{\prime}}{\partial r^{\prime}}+\frac{\lambda^{2} T^{\prime}{ }^{\prime}}{\partial z^{2}}=0
$$


The most general solution of eq. [2.1] is given by

$$
U_{i}^{\prime}=\frac{I \varrho_{1}}{4 \pi} \int_{0}^{\infty}\left\{\bar{K}_{i}(\lambda) e^{-\lambda z}+\bar{\chi}_{i}(\lambda) e^{\lambda z}\right\} J_{\mathrm{o}}(\lambda \cdot r) \mathrm{d} \lambda
$$

where $J_{0}(\lambda r)$ is the Bessel function of first kind and zero order;

$\lambda$ the dummy variable of integration

$\bar{K}_{i}(\lambda)$ and $\bar{\gamma}_{i}(\lambda)$ unknown functions to be determined by applying the boundary conditions.

Therefore the total potential $U_{i}$ is

$$
U_{i}=Y+U^{\prime}{ }_{i}=\frac{I \varrho_{1}}{\dot{4} \pi}\left\lceil\frac{1}{R}+\int_{0}^{\infty}\left\{\bar{K}_{i}(\lambda) e^{-\lambda z}+\bar{\chi}_{i}(\lambda) e^{\lambda z}\right\} J_{o}(\lambda r) \mathrm{d} \lambda\right]
$$

The boundary conditions to be satisfied are given by

$$
\left.\frac{1}{\rho_{1}} \frac{\partial U_{1}}{\partial z}\right|_{z=-z_{0}}=0
$$

i.e. the normal component of the current density vanishes at the airwater discontinuity;

$$
U_{i}=U_{i+1}
$$

i.e. the potential must be continuous at each boundary;

$$
\frac{1}{\varrho_{i}} \frac{\partial U_{i}}{\partial z}=\frac{1}{\varrho_{i+1}} \frac{\partial U_{i+1}}{\partial z}
$$

which establishes the continuity at each boundary of the normal component of the current density;

$$
\left.U_{n}\right|_{z=+\infty}=0
$$

which represents the vanishing of the potential at infinity.

Since we are interested to the potential $U_{1}$ measured with the emitting and receiving electrodes placed horizontally at the bottom 
of the water layer, applying the boundary conditions [2.4] to [2.7] it is obtained ( ${ }^{4}$ )

$$
\begin{gathered}
U_{1}=\frac{I \varrho_{1}}{4 \pi} \int_{0}^{\infty}\left\{1+e^{-2 \lambda h_{1}}+2 K_{1}(\lambda)+K_{1}(\lambda) \mid e^{2 \lambda h_{1}}+\right. \\
\left.\left.+e^{-2 \lambda h_{1}}\right]\right\} J_{o}(\lambda v) \mathrm{d} \lambda
\end{gathered}
$$

being $K_{1}(\lambda)$ the well-known Stefanesco's kernel function appearing in the expression of the potential when the apparatus is at the free surface, given by $\left({ }^{2}\right)$

$$
U_{1}=\frac{I \varrho_{1}}{2 \pi} \int_{0}^{\infty}\left\{1+2 K_{1}(\lambda)\right\} J_{0}(\lambda r) \mathrm{d} \lambda
$$

Eq. [2.8] is more general than eq. [2.9]; in fact putting $h_{1}=0 \mathrm{in}$ eq. [2.8], eq. [2.9] is easily obtained.

The expression of the electric field $E_{1}$, that is measured with the Schlumberger array AMNB at the bottom, can be obtained by differentiating the right-hand member of eq. [2.8] with respect to $r$, and multiplying the derivative by a factor 2 , required to take account that there are two point sources of current, distant $2 r$, which produce contributions to the potential gradient equal to each other both in magnitude and in direction. The result is given by

$$
\begin{gathered}
E_{1}=\frac{I_{\underline{L}}}{2 \pi} \int_{0}^{\infty}\left\{1+e^{-2 \lambda h_{1}}+2 K_{1}(\lambda)+K_{1}(\lambda)\left[{ }_{2}^{2 \lambda h_{1}+}\right.\right. \\
\left.\left.+e^{-2 \lambda h_{1}}\right]\right\} J_{1}(\lambda \cdot) \lambda d \lambda
\end{gathered}
$$

being $J_{1}(\lambda r)$ the Bessel function of first kind and first order.

As is well-known, the apparent resistivity is defined in such a way that it equals always the true resistivity in the case of an homogeneous semispace. When conducting normal superficial Schlumberger soundings on a layered earth, the apparent resistivity is defined as

$$
?_{a}=\left(\pi r^{2}\right) \frac{E}{I}
$$

where $\pi r^{2}$ is the so-called geometrical factor of the array. 
If the same criterion is assumed for defining the apparentresistivity in the case of Schlumberger soundings at the bottom of the water layer, a geometrical factor must be introduced which takes account not only of the electrode arrangement but also of the depth from the surface of the array. In such a way it would result a remarkable and useless complication in establishing the working mathematical expression of the apparent resistivity. In practice it is most convenient to adopt the same geometrical factor $\pi r^{2}$ and introduce the following definition of the apparent resistivity $\bar{D}_{a}$ measured at the bottom

$$
\bar{\varrho}_{a}=\left(\pi r^{2}\right) \frac{E_{1}}{I}
$$

Putting eq. [2.10] into eq. [2.12] we obtain

$$
\begin{gathered}
\bar{\varrho}_{a}=\left.\frac{\varrho_{1}}{2} r \cdot\right|_{0} ^{\infty}\left\{1+e^{-2 \lambda h_{1}}+2 K_{1}(\lambda)+K_{1}(\lambda)\left[3^{2 \lambda h_{1}}+\right.\right. \\
+e^{-2 \lambda h_{1}} \mid\left\{J_{1}(\lambda r) \lambda d \lambda\right.
\end{gathered}
$$

Putting

$\bar{T}_{n}(\lambda)=\frac{\varrho_{1}}{2}\left\{1+e^{-2 \lambda h_{1}}+2 K_{1}(\lambda)+K_{1}(\lambda)\left[\iota^{2 \lambda h_{1}}+e^{-2 \lambda h_{1}}\right]\right\}$

eq. [2.13] rerluces simply to

$$
\overline{\varrho_{a}}=r_{0}^{\infty} \int_{n}(\lambda) J_{1}(\lambda r) \lambda d \lambda
$$

The function $\bar{T}_{n}(\lambda)$ will be called the kernel of the resistivity integral for soundings with the apparatus at the bottom of the water layer.

Before starting in the following section the study of the properties of the kernel $\bar{T}_{n}(\lambda)$, we recall, after Terekhin $\left({ }^{4}\right)$, that the resistivity function $\bar{o}_{a}$ given by eq. [2.13] has the following asymptotical values

$$
\begin{gathered}
\lim _{\left(r / h_{1}\right) \rightarrow 0} \bar{\varrho}_{a}=\frac{\varrho_{1} \varrho_{2}}{\varrho_{1}+\varrho_{2}} \\
\lim _{\left(r / h_{1}\right) \rightarrow+\infty} \bar{\varrho}_{a}=\varrho_{n}
\end{gathered}
$$


3. - The asymptotical values of the hervel function $\bar{T}_{n}$.

In general we may put $\left(^{1}\right)$

$$
K_{1}(\lambda)=\sum_{1}^{\infty}, q_{j} e^{-2 j \lambda \lambda h}
$$

where

$q$ are the so-called coefficients of emission:

$h$ is the maximum common divisor among the thicknesses of all the layers.

Substituting eq. [3.1] into eq. [2.14] and putting $h_{1}=s h$ we get

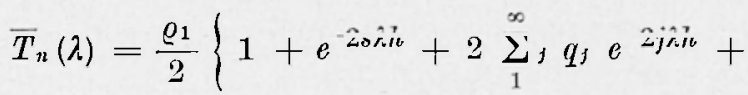

$$
\begin{aligned}
& \left.+\sum_{1}^{\infty} q_{j} e^{2(j-s) \hat{\lambda} n}+\sum_{1}^{\infty} q_{j} e^{-2(j+s) \lambda h}\right\}
\end{aligned}
$$

From eq. [3.2] it can be easily derived that

$$
\lim _{(1 / \lambda \lambda) \rightarrow+\infty} \bar{T}_{n}(\lambda)+\varrho_{1}\left\{1+2 \sum_{1}^{\infty} q_{j}\right\}=\varrho_{n}
$$

and

$$
\lim _{\left(1 / \lambda h_{i}\right) \rightarrow 0} \bar{T}_{n}(\lambda)=\frac{\varrho_{1}}{2}\left\{1+q_{s}\right\}=\frac{\varrho_{1} \underline{\varrho}_{2}}{\varrho_{1}+\varrho_{2}}
$$

Therefore the kernel $\bar{T}_{n}(\lambda)$ has the same asymptotical values as the apparent resistivity $\bar{\varrho}_{a}$ (see eqs. [2.16] and [2.17]) when plotted as a function of $1 / \lambda$, which has the physical dimension of a length.

\section{4. - Calculation of the hernel function $\bar{T}_{n}$ For an $N$-LAYered SECTION}

Let us consider now eq. [2.9] which, as previously mentioned, represents the potential when the apparatus is placed on the free surface. 
Koefoed ( $\left.{ }^{3}\right)$ introduced the kernel function

$$
T_{n}(\lambda)=\varrho_{1}\left\{1+2 K_{1}\left(\lambda_{n}\right)\right\}
$$

and showed that this function satisfies the following recurrent expression

$$
T_{n}(\lambda)=\frac{T_{n-1}(\lambda)+v_{1}}{1+T_{n-1}(\lambda) v_{1} / \varrho_{1}^{2}}
$$

where

$$
v_{1}=\varrho_{1} \frac{1-e^{-2 \lambda h_{1}}}{1+e^{-2 \lambda h_{1}}}
$$

In eq. [4.2] $T_{n-1}(\lambda)$ is the kernel function reduced to the lower boundary plane, i.e. corresponding to a stratigraphic situation in which the first layer is completely suppressed and the measurements are carried out on the new free surface.

From eq. [4.1] we get

$$
K_{1}(\lambda)=\frac{T_{n}(\lambda)}{2 O_{1}}-\frac{1}{2}
$$

Putting eq. [4.4] into eq. [2.14] we have

$$
\bar{T}_{n}(\lambda)=\frac{T_{n}(\lambda)}{4}\left[2+e^{2 \lambda h_{1}}+e^{-2 \lambda h_{1}}\right]+\frac{\varrho_{1}}{4}\left[e^{-2 \lambda h_{1}}-e^{2 \lambda h_{1}}\right]
$$

From eq. [4.3] it is obtained

$$
2^{-2 \lambda h_{1}}=\frac{\varrho_{1}-v_{1}}{\varrho_{1}+v_{1}}
$$

and

$$
2 \lambda h_{1}=\frac{o_{1}+v_{1}}{\varrho_{1}-v_{1}}
$$

With eqs. [4.6] and [4.7], eq. [4.5] becomes

$$
\bar{T}_{n}(\lambda)=\frac{\varrho_{1}^{2}}{\varrho_{1}^{2}-v_{1}^{2}}\left[T_{n}(\lambda)-v_{1}\right]
$$

This last expression is an useful and quite simple formula which relates the kernel $\bar{T}_{n}(\lambda)$, when the apparatus is at the bottom of the 
water layer, to the kernel $T_{n}(\lambda)$ when the apparatus is on the free surface of the same layered model.

If the kernel $T_{n}(\lambda)$ is known for an assigned layered moded, eq. [4.8] allows to construct easily the corresponding kernel $\bar{T}_{n}(\lambda)$. But, in general, $T_{n}(\lambda)$ is neither tabulated nor graphically reported; then it is necessary to construct each time this last function by using the recurrent eq. [4.2] before applying eq. [4.8].

5. APPROXIMATION OF $\bar{T}_{n}$ FOR SMALIEST AND LARGEST VALUES OF $1 / \lambda$

After Toefoed $\left(^{(2-3}\right)$ it can be derived that the kernel $T_{n}(\lambda)$ can be approximated for smallest values of $1 / \lambda$ by the function $T_{2}(\lambda)$, that is to say, by the kernel corresponding with the first two layers. In other words the kernel $T_{n}(\lambda)$ whatever be the number of the layers is asymptotically fitted in its left-hand portion by a two-layer kernel.

In fact eq. [4.1] can be rewritten in the following form

$$
T_{n}(\lambda)=\varrho_{1}\left\{\frac{1+K_{1}(\lambda) /\left[1+K_{1}(\lambda)\right]}{1-K_{1}(\lambda) /\left[1+K_{1}(\lambda)\right]}\right\}
$$

The function $K_{1}(\lambda) / 1+K_{1}(\lambda)$ is asymptotical for smallest values of $1 / \lambda$ to the function $\left({ }^{2}\right)$

$$
\eta_{1}(\lambda)=\frac{\varrho_{2}--\varrho_{1}}{\varrho_{2}+\varrho_{1}} e^{-2 \lambda h_{1}}
$$

Putting eq. [5.2] into eq. [5.1] we have

$$
T_{n}(\lambda) \approx \varrho_{1} \frac{1+y_{1}(\lambda)}{1-y_{1}(\lambda)}=T_{2}(\lambda)
$$

With eq. [5.3], eq. [4.8] becomes

$$
\bar{T}_{n}(\lambda) \approx \frac{\varrho_{1}{ }^{2}}{\varrho_{1}{ }^{2}-v_{1}{ }^{2}}\left[T_{2}(\lambda)-v_{1}\right]=\bar{T}_{2}(\lambda)
$$

Eq. [5.4] shows that also the kernel $\bar{T}_{n}(\lambda)$, whatever be the number of the layers, is asymptotically fitted in the left-hand portion by a two-layer kernel $\overline{T_{2}}(\lambda)$.

From eq. [4.3] we have that for largest values of $1 / \lambda$, in comparison with the thickness $h_{1}$ of the water layer, the function $v_{1}$ becomes increasingly small so that it can be neglected in eq. [4.8]: it follows that

$$
\bar{T}_{n}(\lambda) \approx T_{n}(\lambda)
$$


Eq. [5.5] shows that the kernel $\bar{T}_{n}(\lambda)$, when the apparatus is at the bottom of the water layer, will not differ from the value of the kernel $T_{n}(\lambda)$, which would be obtained when the same apparatus is at the free surface, for largest values of $1 / \lambda$.

These important properties of the kernel $\bar{T}_{n}$ will be essential for the process of the direct interpretation of resistivity soundings with the apparatus at the bottom.

6. - TIIEORETICAL COMPARISON BETWEeN THE BOTTOM AND FREE SURFACE METHODS

A) The two-layer case.

In fig. 2 a set of two-layer curves are shown for $\bar{T}_{2}\left(\lambda_{)}\right) / \varrho_{1}$ against $1 / \lambda h_{1}$ with varying values of the ratio $\varrho_{2} / \varrho_{1}$. These curves can be compared with those of fig. 3 representing the function $T_{2}(\lambda) / \varrho_{1}$ against $1 / \lambda h_{1}$ for the same ratios $\varrho_{2} / \varrho_{1}$.

The following differences can be noticed:

a) the curves of $\bar{T}_{2} / \varrho_{1}$ are all of ascending type. This is a normal consequence of the asymptotical behaviour studied in section 3;

b) the same curves have no common asymptote;

c) when $\varrho_{2} / \varrho_{1} \rightarrow 0$ the $\bar{T}_{2} / \varrho_{1}$ curves become flatter and flatter so that a distinction in the trend of two contiguous curves becomes more and more imperceptible;

d) when $\rho_{2} / \varrho_{1} \rightarrow+\infty$ the curves approach in the trend the corresponding of fig. 3 . In other words this new technique does not add any new information to those obtained with the array on the free surface;

$e$ ) for intermediate values of $\varrho_{2} / \varrho_{1}$ (in the case of fig. 2, for $0.5 \leqslant o_{2} / o_{1} \leqslant 10$ ) the new curves clearly show a wider width between the left and right extreme values. In this case the new technique presents a greater resolving power. 


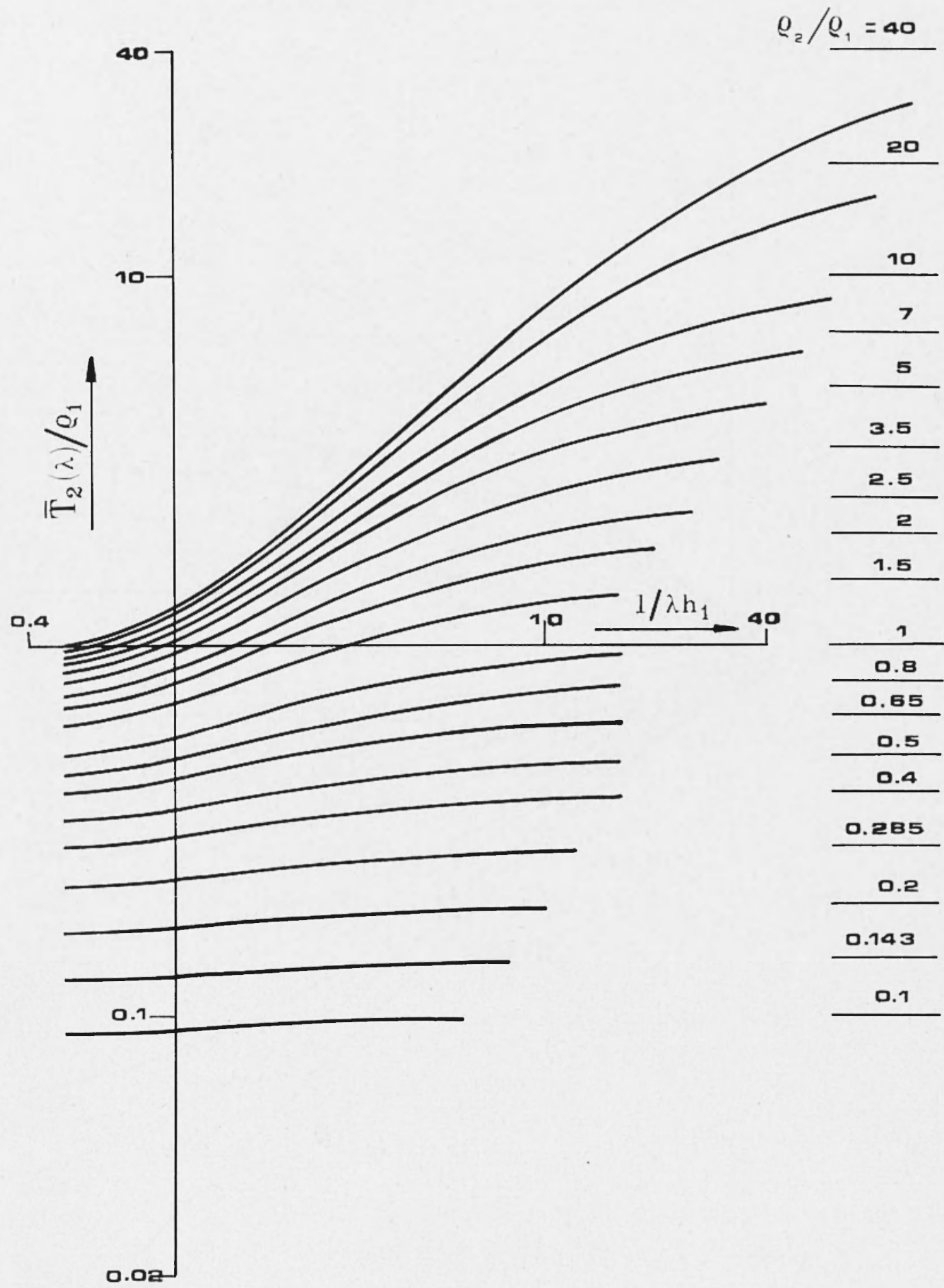

Fig. 2 - A set of two-layer curves for the water-bottom kernel function $T_{2}(\lambda)$ for various resistivity contrasts. 


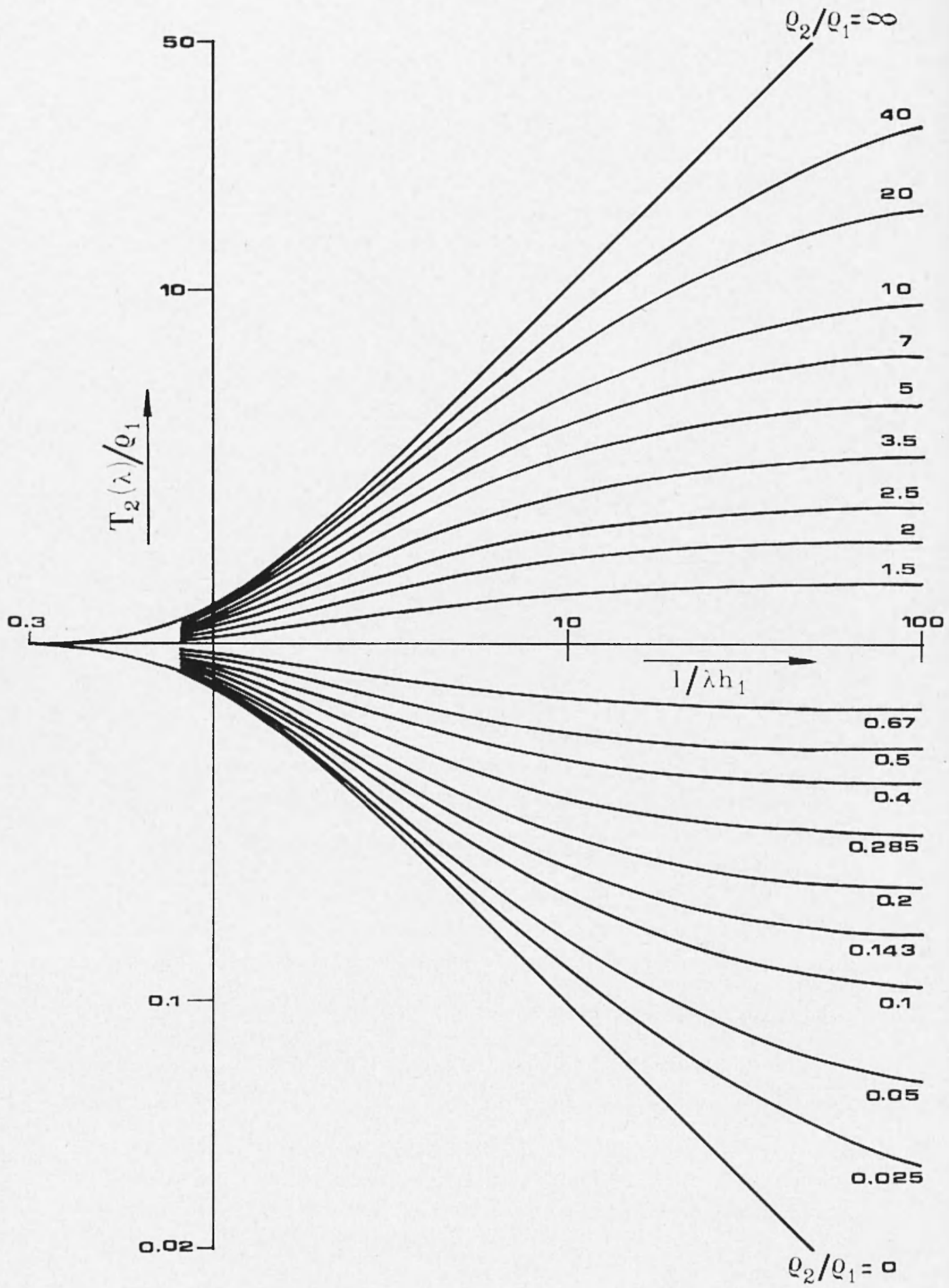

Fig. 3 - A set of two-layer curves for the free surface kernel function $T_{2}(\lambda)$ for various resistivity contrasts. 
B) The three-layer case.

Now we compare the theoretical curves of $\bar{T}_{3} / \underline{O}_{1}$ and $T_{3} / o_{1}$, both as function of $1 / \lambda \mu_{1}$, for the four foundamental three-layer sections:

I) $K$-type, or maximum type $\left(\varrho_{1}<\varrho_{2}>\varrho_{3}\right)$;

II) $H$-type, or minimum type $\left(\varrho_{1}>\varrho_{2}<\varrho_{3}\right)$;

III) $A$-type, or double ascending type $\left(\varrho_{1}<\varrho_{2}<\varrho_{3}\right)$;

IV) $Q$-type, or double descending type $\left(\varrho_{1}>\varrho_{2}>\varrho_{3}\right)$.

\section{Case I)}

The following layering parameters have been choosen for the resistivities:

$$
\varrho_{2} / \varrho_{1}=1.5 ; \quad \varrho_{3} / \varrho_{1}=0.2 .
$$

The values of the ratio $h_{2} / h_{1}$ are: $0.1 ; 0.2 ; 0.3 ; 0.5 ; 0.7 ; 1 ; 2 ; 3 ; 5$; $10 ; 15 ; 25$.

In the upper part of fig. 4 there is a set of curves $\bar{T}_{3} / \varrho_{1}$ and in the lower $T_{3} / \varrho_{1}$. The presence of the maximum is remarkably more pronounced in the former set of curves than in the latter.

\section{Case II)}

In fig. 5 the curves of $\bar{T}_{3} / \varrho_{1}$ and $T_{3} / \varrho_{1}$ are reported for the ratios:

$$
\varrho_{2} / \varrho_{1}=0.65 ; \varrho_{3} / \varrho_{1}=100 .
$$

and with the same values of $h_{2} / h_{1}$ as in the case I).

In this case the new curves have no minimum and are of double ascending type. This is a consequence of the always ascending trend of the two-layer curves.

\section{Case III)}

Fig. 6 shows the same two functions for

$$
\varrho_{2} / \varrho_{1}=1.5 ; \quad \varrho_{3} / \varrho_{1}=100 .
$$



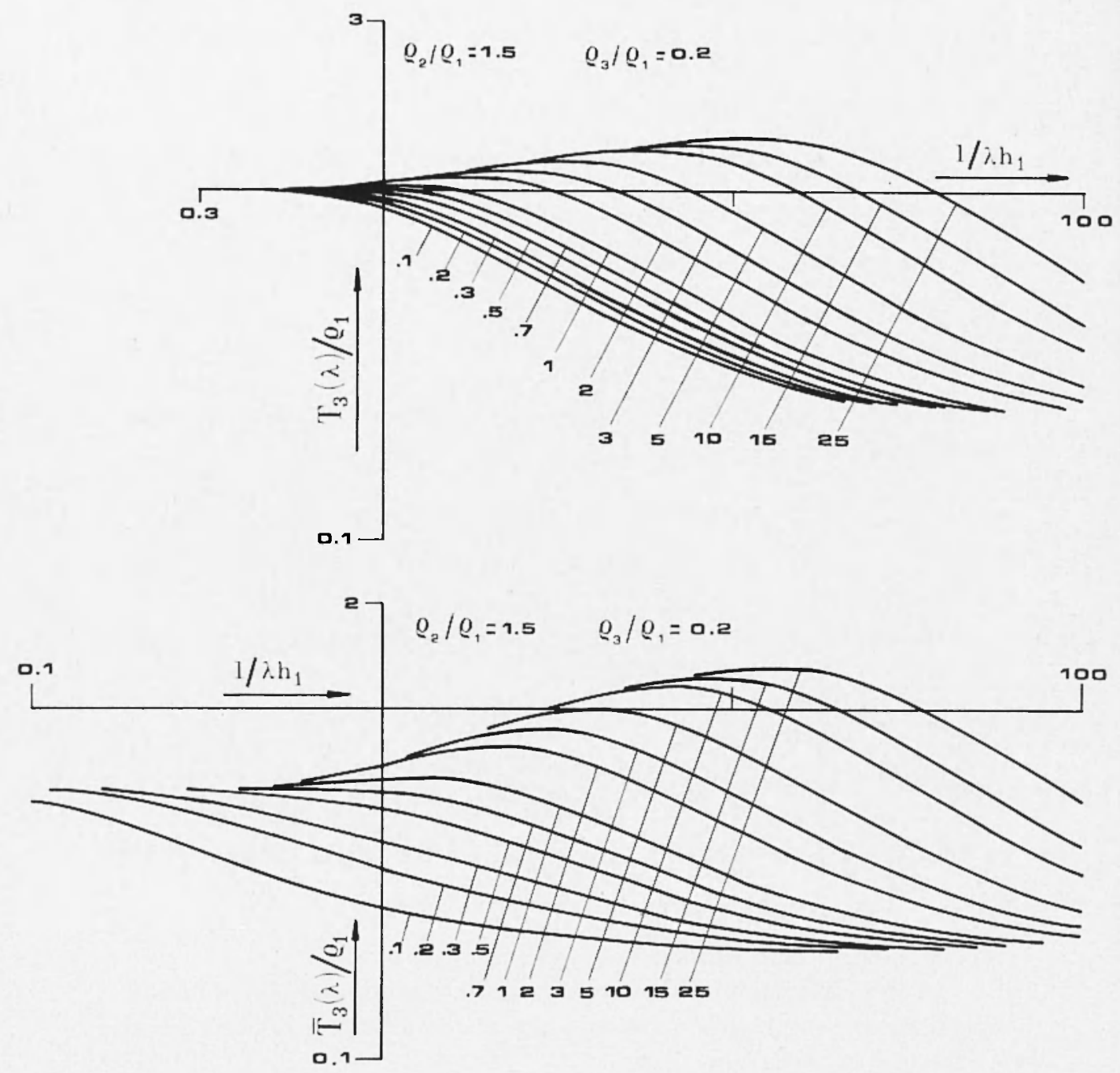

Iig. 4 - Comparison between the free surface and water-bottom kernel functions in the case of a $K$-type three-layer section. Parametric values for each curve are the values of the ratio $h_{2} / h_{1}$. 

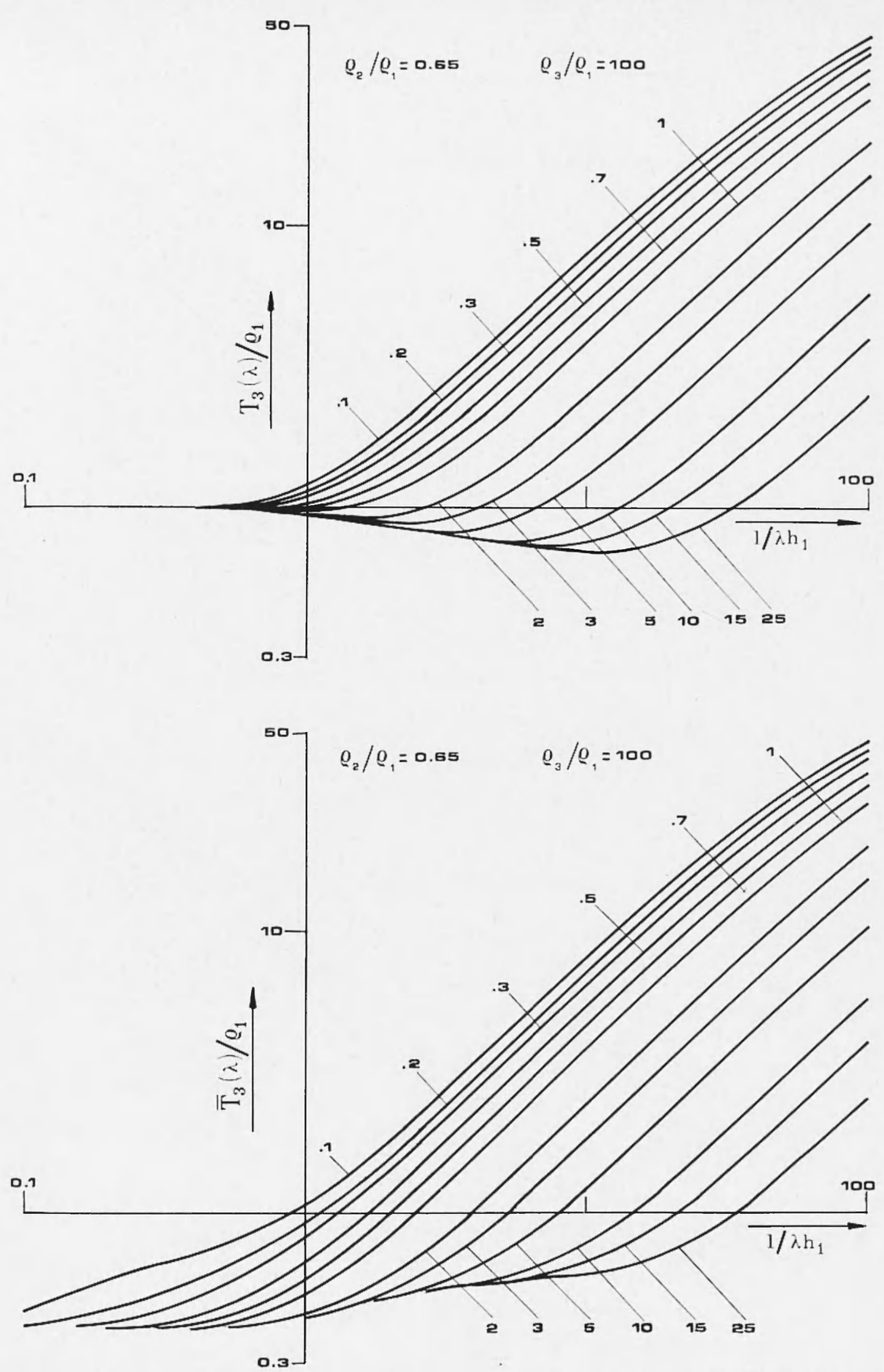

Fig. 5 - Comparison between the free surface and water-bottom kernel functions in the case of an $H$-type three-layer section. Parametric values for each curve are the values of the ratio $h_{2} / h_{1}$. 

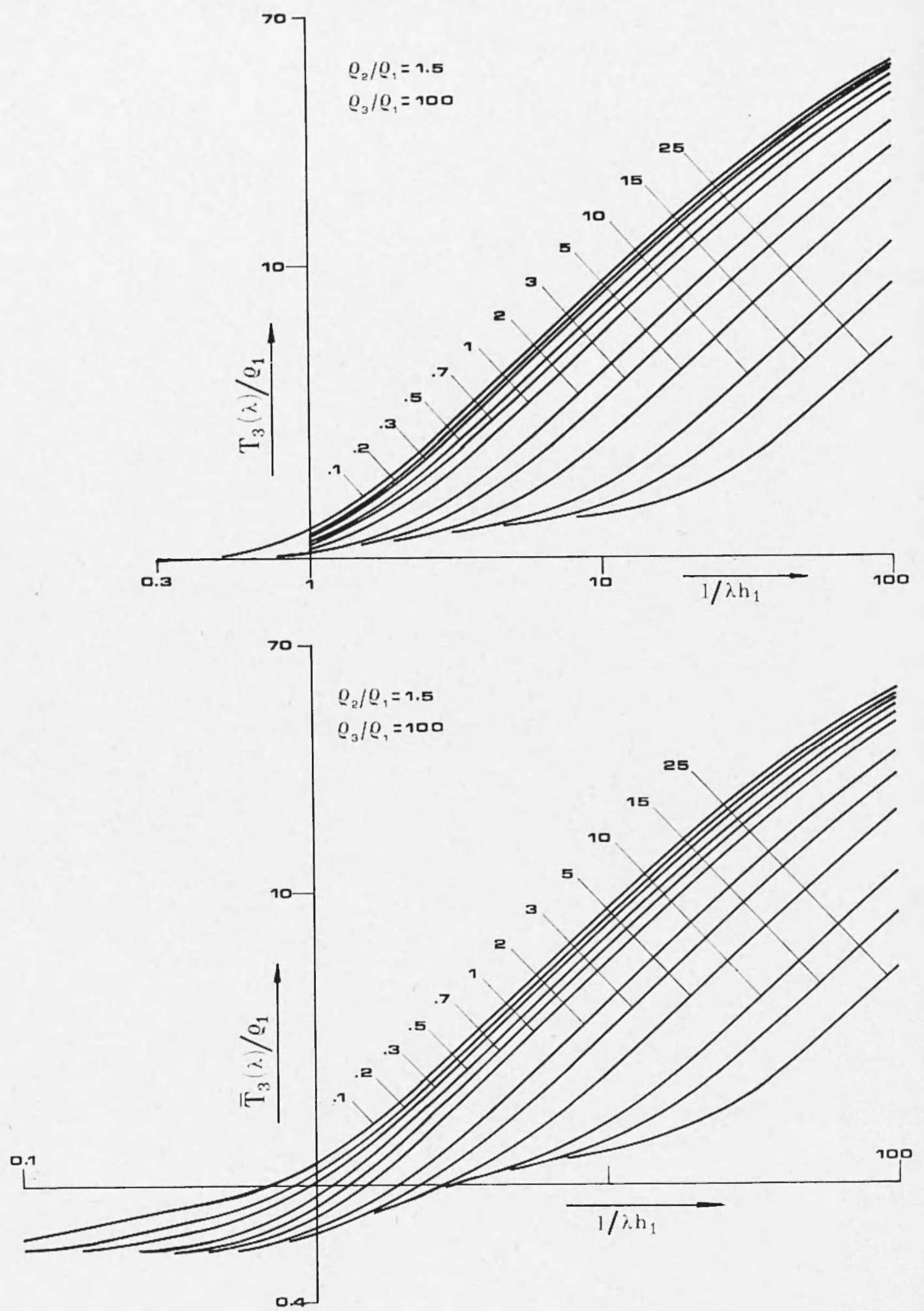

Fig. 6 - Comparison between the free surface and water-bottom kernel functions in the case of an $A$-type three-layer section. Parametric values for each curve are the values of the ratio $h_{2} / h_{1}$. 
The double ascending trend in the new curves is preserved and more pronounced.

\section{Case IV)}

Finally in fig. 7 the two sets of curves are shown for the ratios

$$
\varrho_{2} / \varrho_{1}=0.65 ; \quad \varrho_{3} / \varrho_{1}=0.2 .
$$

The double descending characteristic is now lost and the new curves present a well marked maximum.

By analysing and comparing all the sets of curves the following conclusions can be drawn:

a) The new curves are of only two different types as concerns their general shape:

- with a maximum which includes the old $K$ and Q-types

- of double ascending type which includes the old $H$ and $A$-types.

This fact does not constitute a restriction but it is a normal consequence of the asymptotical behaviour.

b) A greater resolving power is clearly evidenced in all the new curves, especially for the littlest values of the ratio $h_{2} / h_{1}$ where the curves of $T_{3} / O_{1}$ strongly crowd together. The left-hand portion of these new curves shows still more markedly such important and useful property.

c) In the case of the curves with a maximum, corresponding to $K$ and $Q$-type curves for $T_{3} / \varrho_{1}$, the suppression principle is completely overcome. In fact a sight on the $T_{3} / \varrho_{1}$ curves when $h_{2} / h_{1}$ becomes increasingly smaller show that these curves can be confused with twolayer curves. This is not the case for the curves of $\bar{T}_{3} / \varrho_{1}$, since, as we have previously pointed out, the two-layer curves are always of ascending type and therefore there will never be any ambiguity.

d) In the case of double ascending curves, corresponding to $H$ and $A$-type curves for $T_{3} / \varrho_{1}$, the suppression principle is less markedly overcome than case $e$ ). Also in this case the $T_{3} / o_{1}$ curves for smallest values of $h_{2} / h_{1}$ can be confused with two-layer curves. The $\bar{T}_{3} / \varrho_{1}$ 


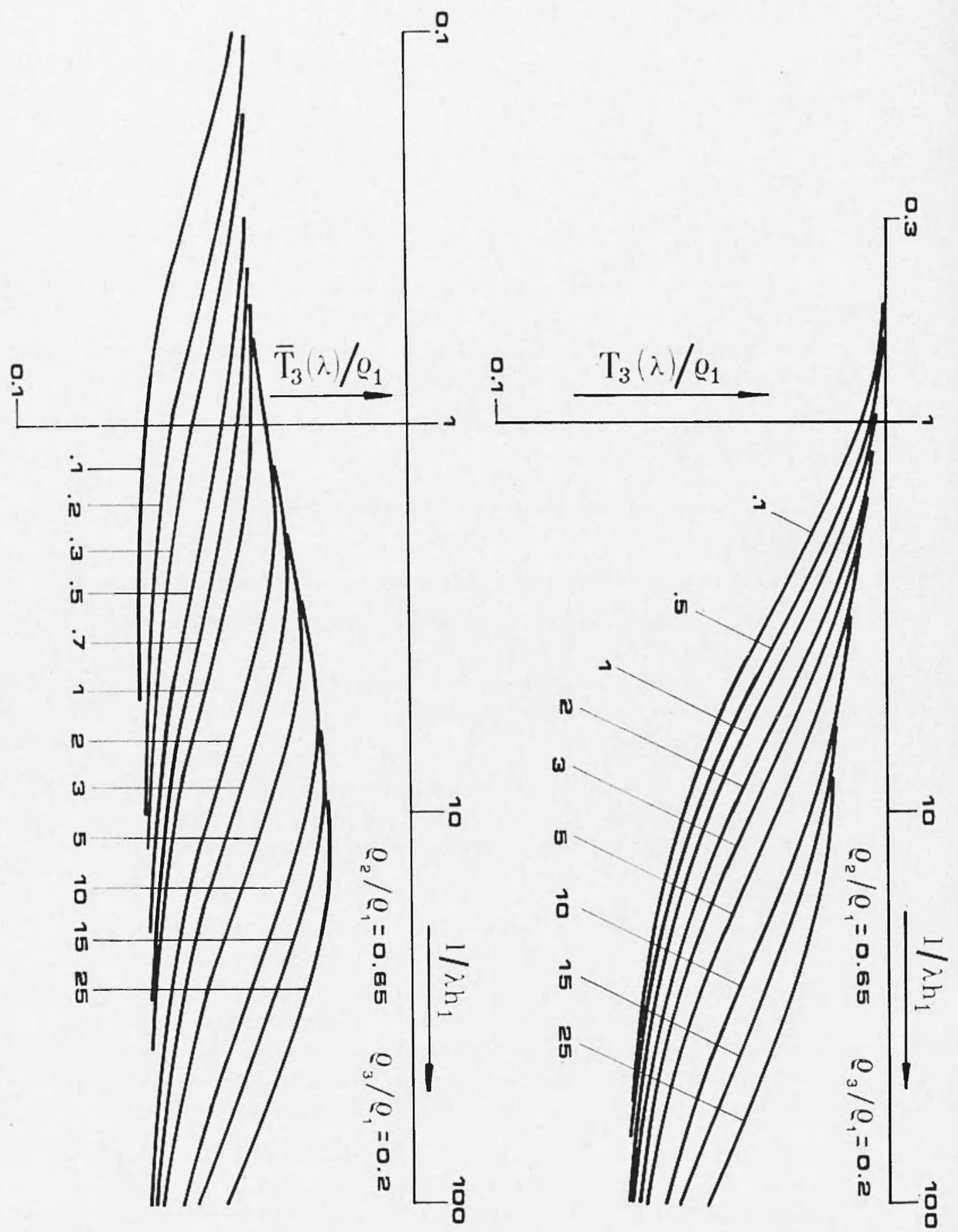

Fig. 7 - Comparison between the free surface and water-bottom kernel functions in the case of a $Q$-type three-layer section. Parametric values for each curve are the values of the ratio $h_{2} / h_{1}$. 
curves for $h_{2} / h_{1} \leqslant 0.3$ show a visible hump in the extreme left-hand portion which becomes increasingly pronounced as $h_{2} / h_{1} \rightarrow 0$, so that also in this case there will never be any ambiguity.

\section{7. - CONCLUdING REMARKS}

From the above analysis that has been for simplicity restricted to two and three-layer sections, but that can be easily expanded to more layers, the following conclusions seem to be of greatest interest:

a) as concerns the study of the properties of the kernel function, its treatment is mathematically simple so allowing to construct the model curve for any desired $n$-layered section without the use of electronic computers as is the case of the apparent resistivity curves.

b) Regarding the features of the water bottom method, it shows, when adopted in opportune situations, many advantages as concerns the resolving power and improvements are gained in eliminating the ambiguities imposed by the principle of suppression.

\section{REFERENCES}

(1) Bhattacharya P. K. \& Patra H. P., 1968. - Direct current geoelectric sounding. Elsevier Publishing Co., Amsterdam.

(2) Kosfosd O., 1968. - The application of the kernel function in interpreting geoelectrical resistivity measurements. "Geoexploration Monographs", Series 1, No. 2. Gebruder Borntraeger, Berlin.

(3) KoEFoed 0., 1970. - .1 fast method for determining the layer distribution from the raised kernel junction in geoelectrical sounding. "Geophysical Prospecting", XVIII, 4.

(4) Terekmix E. I., 1902. - Theoretical bases of electrical probing with an. apparatus immersed in water. In N. RAsT (Editor), "Applied Geophy. sics, U.S.S.R.", Pergamon Press, London.

(5) VAN'YAN L. L., 1956. - Theoretical curves for electrical sea probing with a sea-bottom apparatus. "Applied Geophysics", 50, Gostoptekhizdat. (in Russian). 